\title{
Astrophysical Applications of Delta Scuti Stars
}

\author{
Michel Breger \\ Institut für Astronomie, Universität Wien, Türkenschanzstr. 17, \\ A-1180 Wien, Austria
}

\begin{abstract}
The $\delta$ Scuti stars pulsate with a large number of low-order radial and nonradial $p$-modes. There also exists some evidence for the presence of $g$-modes.

The pulsational variability outside the classical $\delta$ Scuti star instability strip (A1V - F0V on the main sequence) is examined. New measurements do not confirm the short-period variability of the hot B9p star ET And. However, a number of cool, early-F stars shows variability around one day: the $\gamma$ Dor variables. An explanation involving nonradial $g$ modes appears the most promising.

An examination of available information on the multi-periodicity of $\delta$ Scuti stars shows no systematic behaviour in which pulsation modes are excited to an observable level with luminosity and temperature. The asteroseismological potential of $\delta$ Scuti variables is briefly examined.

So far, the period changes of five luminous Pop. I $\delta$ Scuti variables have been studied. While stellar evolution predicts period increases, decreases have been observed and reported for all five stars. The statistical significance of this result and alternative explanations are briefly examined.
\end{abstract}

\section{Introduction}

The extension of the Cepheid Instability Strip crosses the main sequence among the $A$ and $F$-type stars in a $1000 \mathrm{~K}$ wide band. The $\delta$ Scuti variables are shortperiod pulsators situated on and near the main sequence inside the strip with periods from one half to eight hours. Among stars of Pop. I, the amplitudes of pulsation are usually small with visual amplitudes of a few mmag, but can be as large as 800 mmag.

The position of the $\delta$ Scuti stars of Pop. I in the Hertzsprung-Russell Diagram is shown in Fig. 1. Stars with uncertain absolute magnitudes or uncertain variability were rejected for this plot. The edges of the instability strip are drawn to represent the observed distribution of stars with photometrically detectable short-period variability.

While the Sun shows thousands of detectable pulsation modes, the maximum number of modes detected so far for individual $\delta$ Scuti stars is only around 15. An attractive aspect of the study of $\delta$ Scuti stars is the wide range of the stellar evolutionary stages of these stars, ranging from pre-main-sequence through hydrogen burning on the main sequence stage to the giant stage. 


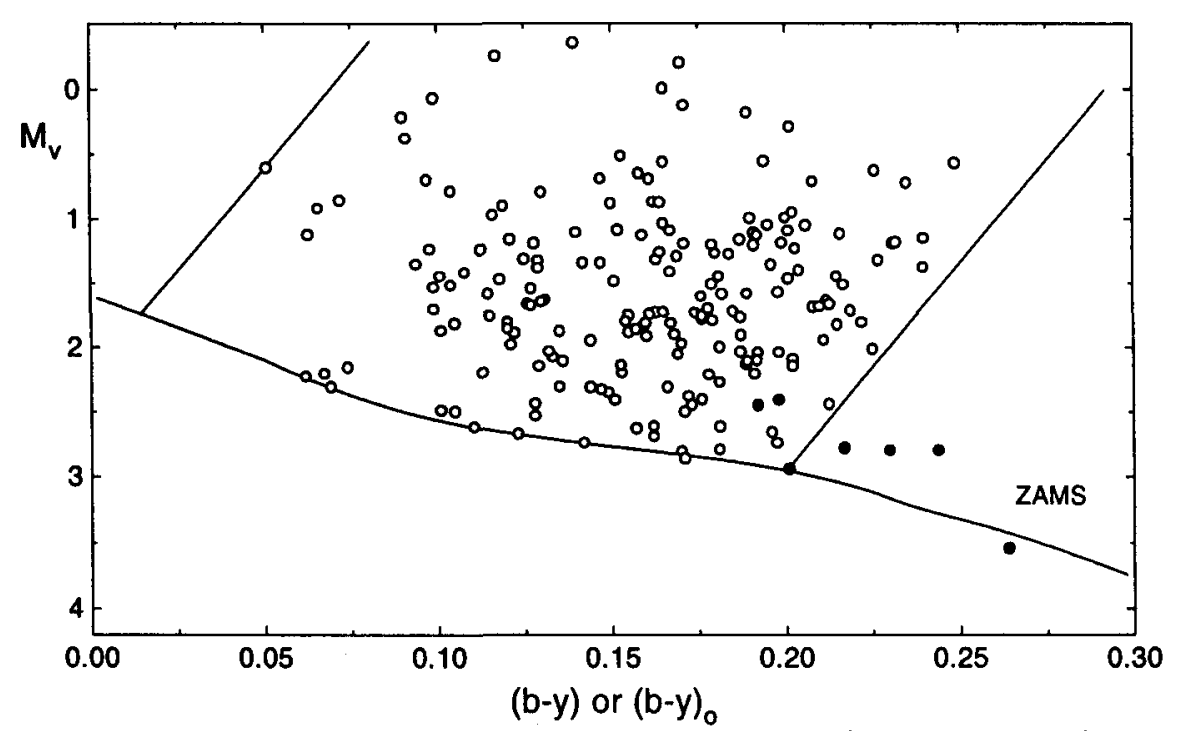

Figure 1. Position of the Pop. I $\delta$ Scuti (open circles) and the related $\gamma$ Dor variables (closed circles) in the HR diagram.

\section{Pulsation outside the classical borders of the instability strip}

The Lower Instability Strip contains several different related types of pulsators: the Pop. I $\delta$ Scuti stars (radial and nonradial low-order $p$-modes), the Pop. II $\delta$ Scuti stars (also called SX Phe stars, radial modes with large amplitudes) and the roAp stars (magnetic stars, high-order $p$-modes).

\subsection{ET And and the Maia variables}

Pulsation beyond the hot instability strip border, in the region bordered by the $\delta$ Scuti stars and the $53 \mathrm{Per} / \mathrm{SPB}$ variables (probably $g$ modes) has been suspected many times (e.g., Struve 1955, Lehmann et al. 1995) but not confirmed. Some inconclusive studies of these mythical Maia variables are available. If the reported variability were real, the period lengths would indicate radial or nonradial $p$-mode pulsation, as in the $\delta$ Scuti stars.

In recent years, the most often quoted example of a short-period, hot pulsator has been the B9p(Si) star ET And. This star was suspected by Hildebrandt, Schöneich \& Lange (1985) and Kuschnig et al. (1990) to show $\delta$ Scuti-like variability of $0.10 \mathrm{~d}$ and a peak-to-peak amplitude of about $4 \mathrm{mmag}$ in $B$. This variability would be superposed on the $1.6 \mathrm{~d}$ variations caused by the rotation of this peculiar Si star. An interpretation of the rapid variations in terms of p-modes would suggest that the hot border of the instability strip would need to be shifted considerably towards hotter temperatures of $11500 \mathrm{~K}$. However, a two-week multi-site campaign undertaken during 1994 September coordinated by R. Kuschnig and W. Weiss showed the short-period constancy of ET And and 
identified a previously used comparison star as the variable. With this discovery of constancy, the Maia variables return to their mythical status.

\subsection{The $\gamma$ Dor variables}

The variability of a number of stars on or beyond the cool border of the traditional $\delta$ Scuti instability strip (see Fig. 1) is more convincing. This variability is unusual enough so that a different type of variable might be involved: the periods are too long for $\delta$ Scuti-like $p$-mode pulsation.

Burke, Burke \& Lady (1977) noticed that the F2V star, HD 164615, varied photometrically with a period of $0.815 \mathrm{~d}$ and an amplitude of 0.05 mag. Abt, Bollinger \& Burke (1983) call this star a probable spotted single F-type star. The variability would be produced by the spot rotating with the star. They rule out ellipsoidal variability on the basis of constant radial velocity (measured scatter of $\pm 1.6 \mathrm{~km} \mathrm{~s}^{-1}$ compared to an error of $\pm 2.0 \mathrm{~km} \mathrm{~s}^{-1}$ ).

A number of other variable early $F$ stars with characteristic periods of variation $\sim 1 \mathrm{~d}$ have been proposed: the star $\gamma$ Dor (Cousins 1966, Balona, Krisciunas \& Cousins 1994), 9 Aur (Krisciunas \& Guinan 1990), HD 224638 and HD 224945 (Mantegazza, Poretti \& Zerbi 1994), GM Com (Handler 1994), and possibly HR 1459 (Krisciunas, private communication). The star HD 96008 $(\mathrm{P}=0.31 \mathrm{~d}$, Matthews 1990) probably is an ellipsoidal variable (Mantegazza, Poretti \& Zerbi 1994) and is omitted here. While the determination of periods in the $1 \mathrm{~d}$ range is difficult at best, the situation is made even more complicated by the possible existence of double waves in the light curves.

Three explanations have been proposed:

1. Close double stars: this explanation is unlikely because of the need to match the observed $v \sin i$ values with the radial velocity amplitudes. The derived mass functions are improbably small. We refer to the discussions by Balona, Krisciunas \& Cousins (1994) concerning the star $\gamma$ Dor and Abt, Bollinger \& Burke (1983) for HD 164615.

2. Surface spots: this explanation is attractive because it can explain the small radial velocity changes, the double-wave behaviour of the light curve (two or more spots) and even the existence of two close periods (differential rotation). The remaining light curve irregularities could be explained by the formation and destruction of the spots and/or by their migration. The periods of variation are compatible with the expected rotation periods of the stars. However, the origin of such spots in such relatively hot stars is difficult to explain, especially if one considers the dynamo mechanism which requires a deep convection layer.

3. Nonradial $g$-mode pulsation: The discovery of three periods in $\gamma$ Dor (Balona, Krisciunas \& Cousins 1994) of 0.757, 0.733 and $0.678 \mathrm{~d}$, respectively, as well as the line-profile variations, are best explained by nonradial pulsation. These relatively long periods cannot be matched by radial or $p$-mode pulsation and would require $\mathrm{g}$ modes. Detailed models for $g$-mode pulsators should test whether the observed small or zero radial velocity changes observed for some stars might be a problem. 
At this point, explanations involving $g$-mode pulsations seem to be the most attractive. However, the study of these $\gamma$ Dor variables is still in its infancy and may offer many surprises in the future.

\section{Asteroseismology of $\delta$ Scuti stars}

The rich mixture of simultaneously excited radial and nonradial $p$-modes in many Pop. I $\delta$ Scuti stars makes them an ideal tool for asteroseismological studies. The excited $p$-modes are of low order, viz. $0 \leq \mathrm{n} \leq 7$. Modes of higher orders have not yet been found, e.g., a WET/DSN campaign to look for these modes in the star FG Vir could not photometrically detect any (Breger et al. 1995). For low-order modes, the asymptotic theory of frequency spacing does not hold, so that we have to compare the observed frequency spacings with pulsation models computed specifically for the star studied.

One of the most challenging and promising aspects of $\delta$ Scuti star asteroseismology concerns the modes with mixed character: low-order $p$-modes may develop $g$-mode type behaviour in the interior (see Dziembowski \& Krolikowska 1990, Dziembowski \& Goode 1992). This means that the study of the observed $p$-mode spectrum is a probe of the stellar interior. The trapped modes may even provide an asteroseismological test for convective overshooting theories (Dziembowski \& Pamyatnykh 1991). An additional probe of the stellar structure is provided by observing the frequency spacing of rotationally split modes:

$$
\sigma_{\mathrm{m}}=\sigma_{0}+\left(\mathrm{C}_{\mathrm{L}}-1\right) \mathrm{m} \Omega+\mathrm{D}_{\mathrm{L}} \mathrm{m}^{2} \Omega^{2} / \sigma_{0}
$$

where $\sigma$ represents the frequency of pulsation of the mode with azimuthal quantum number $m, \Omega$ is the rotational frequency, while $C_{L}, D_{L}$ are coefficients depending on the model and pulsation mode (see Dziembowski \& Goode 1992).

An example of the methods which can be applied can be found in Goupil et al. (1993), who match the five observed frequencies of GX Peg with models and examine whether the star rotates as a solid body.

Not all $\mathrm{m}$ values are excited at the same time to an observable amplitude, so that the observed frequency spectra of $\delta$ Scuti stars usually do not show complete patterns of rotational splitting of successive radial orders. The missing frequencies make the identification of the observed frequencies with pulsation modes difficult. A large number of reliable mode identifications for each star are usually required to put constraints on models of stellar structure and evolution. Consequently, we urge that the highest priority should be given to increase the number and reliability of the frequencies for individual stars. Some of the modes studied have amplitudes just below 1 mmag: lengthy multi-site campaigns involving high-precision photometry are needed.

Several astronomical teams are concentrating on multi-site campaigns of individual stars. Some of these are:

1. Delta Scuti Network (DSN), e.g., Breger et al. (1989),

2. a group at Merate Observatory, e.g., Mantegazza \& Poretti (1992), 
3. STEPHI Network, e.g., Belmonte et al. (1994),

4. STACC, e.g., Frandsen et al. (1994),

5. Whole Earth Telescope (WET), e.g., Handler (1995).

What is the status of our knowledge of multiple pulsation modes of $\delta$ Scuti stars? Very few stars have been studied in detail and we have selected the most promising candidates for asteroseismology from data of hundreds of stars in the literature. The criteria by inclusion in the present group are:

1. The number of reliably detected frequencies must be $\geq 4$,

2. Information of reasonable quality on the basic stellar parameters such as $T_{\text {eff }}, \log g$ and $M_{\mathrm{V}}$ must exist.

3. Stars with variable amplitudes were included only when the variability was judged to be intrinsic to the star rather than caused by incomplete multi-frequency solutions. For variable amplitudes an average amplitude was calculated for each frequency.

The results are presented in Figs. 2 and 3 , where the stars have been ordered in decreasing temperature. To correct for the different stellar radii, the frequency scales for the individual stars have been normalised by the value of the pulsation constant, $Q$. This makes it possible to show the predicted values for the four low-order radial modes. The observationally determined $Q$ values are uncertain to about $18 \%$ even for stars with excellent $u v b y \beta$ values (see Breger 1990a). Consequently, small horizontal shifts are still possible.

Most of the stars shown in the diagrams are not radial pulsators. Nevertheless, since the frequency separations between successive radial orders of nonradial modes are similar to those of radial modes, the lines drawn can be an important diagnostic tool for mode identification.

The following stars were selected: CD -24 7599 , FM Com (Paparo et al. 1993, Bax, Wehlau \& Sharpe 1995), $\theta^{2}$ Tau (Breger et al. 1989), GX Peg (Michel et al. 1992), BU Cnc (Breger et al. 1993, Belmonte et al. 1994), BN Cnc (Belmonte et al. 1994, Kovacs 1981), UU Ari (Ostermann et al. 1991), FG Vir (Breger et al. 1995), 21 Mon (Stobie et al. 1977), AI Vel (Walraven, Walraven \& Balona 1992), HD 18878 (Mantegazza \& Poretti 1993), HD 224639 (Mantegazza, Poretti \& Zerbi 1995), BI CMi (Mantegazza \& Poretti 1994), X Cae (Mantegazza \& Poretti 1992), 4 CVn (Breger 1990b), 44 Tau (Poretti et al. 1993).

Surprisingly, Figs. 2 and 3 do not show any systematic behaviour of the excited pulsation modes with temperature and luminosity. The astrophysical reasons for mode selection by the stars must be regarded as presently unknown.

\section{Period variability and evolution}

The short periods of $\delta$ Scuti stars allow the observational available determination of the period changes expected from stellar evolution within a few decades. The 


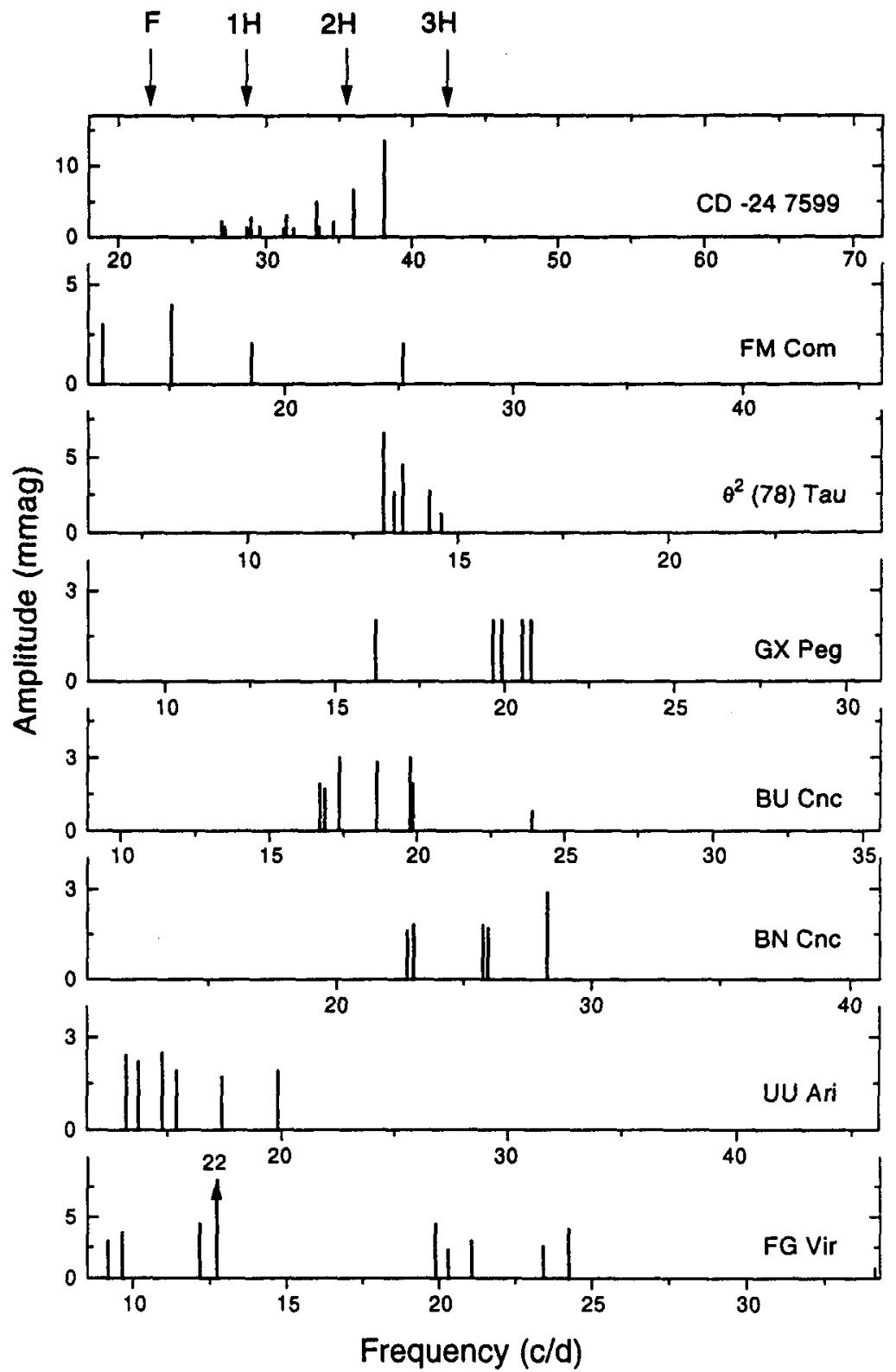

Figure 2. Multiple pulsation modes detected for well-studied $\delta$ Scuti stars. The stars have been ordered in decreasing temperature. For each star, the frequency scale has been normalized by the value of the pulsation constant, $Q$. 


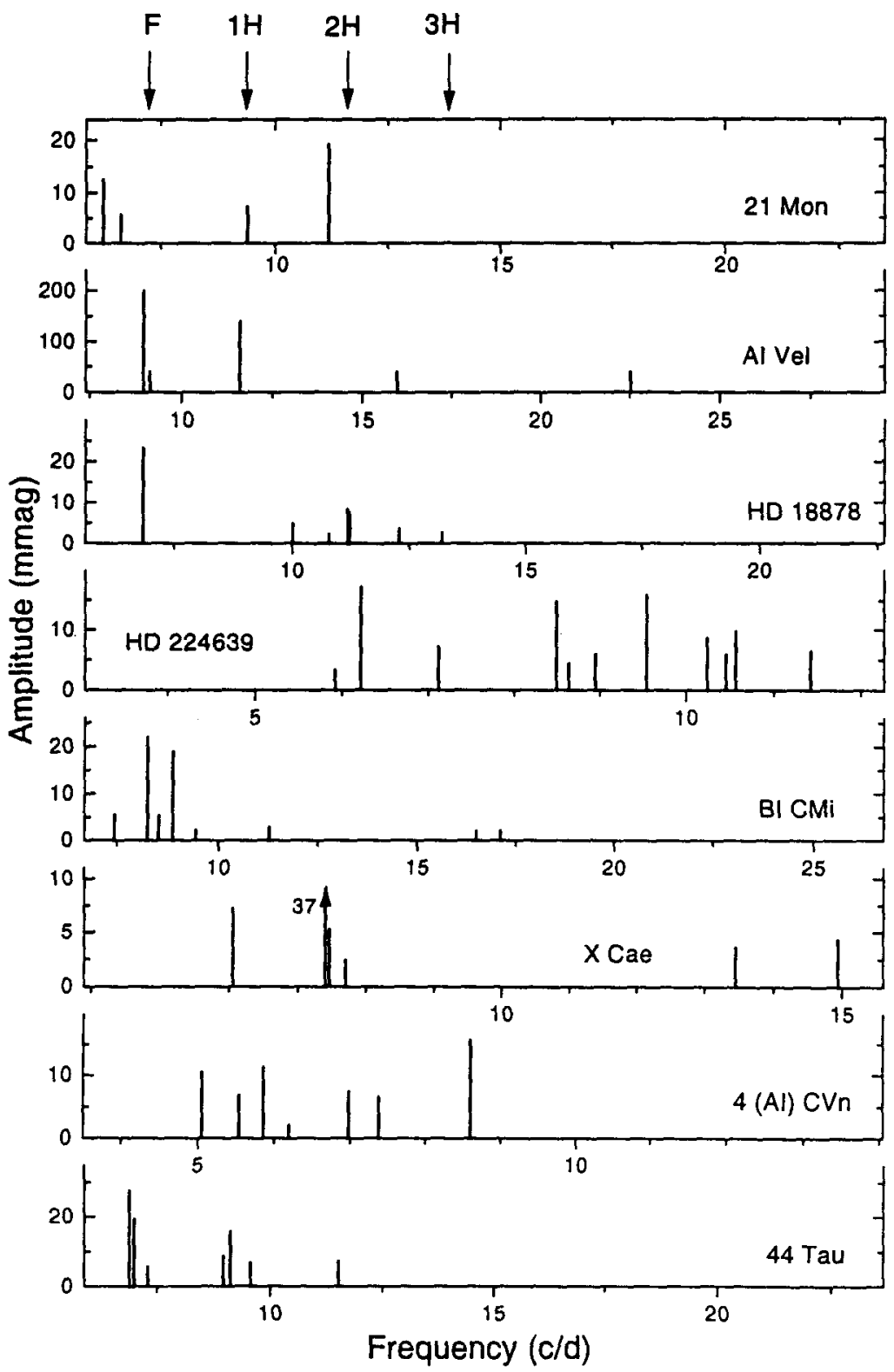

Figure 3. Continuation of Figure 2. In the figure, the theoretically predicted frequencies for low-order radial modes are shown for comparison. Note the lack of systematics of the detected pulsation modes with temperature and luminosity. 
available information was summarised during a previous conference of this series (Breger 1990c). A surprising result was the fact that the four Pop.I $\delta$ Scuti giants studied so far with $\mathrm{P} \geq 0.13 \mathrm{~d}$ (DY Her, VZ Cnc, BS Aqr, $4 \mathrm{CVn}$ ) all show decreasing periods. Stellar evolution theory, however, predicts increasing periods in that part of the Hertzsprung-Russell Diagram due to increasing radii. An attempt by Guzik \& Cox (1991) to explain the period decreases on the basis of helium diffusion was unsuccessful.

One difficulty is that the conclusion of period decreases is based on only four stars. An important observational update is now provided by Rodriguez et al. (1995). They confirm the previous conclusions with new data and also report a period decrease for a fifth $\delta$ Scuti giant, i.e., $(1 / \mathrm{P}) \mathrm{dP} / \mathrm{dt}=-10.610^{-8}$ for RS Gru.

The disagreement with stellar evolution theory, therefore, still exists. However, one should consider the possibility that observed period changes could be caused by other effects, such as period readjustments caused by discrete mixing events inside the star (Sweigart \& Renzini 1979). Such changes would be of either sign. This hypothesis might also explain why period changes could be sudden, rather than continuous. The difficulty with this explanation lies in the fact that the observed period decreases seem to be systematic: positive in the lower part of the HRD and negative in the cool luminous part.

The dilemma might be solved if the available measurements were of insufficient quality and quantity. The situation can be illustrated with one of the better determined stars, DY Her. When the excellent analysis of the times of maxima by Szeidl \& Mahdy (1980) is supplemented by more recent results by Yang et al. (1993), the derived period decrease is lowered by about 30\%! Furthermore, it is not clear whether the changes are smooth or discontinuous.

Let us look at another $\delta$ Scuti giant, VZ Cnc. Percy, Matthews \& Wade (1980) as well as Cao \& Jiang (1992) have reported that the period of VZ Cnc is decreasing with a statistical significances of three standard deviations. On the other hand, a different analysis of the new and old times of maximum by Arellano Ferro et al. (1994) led them to interpret the variations in in terms of a binary light-time effect (sinusoidal variations). An inspection of the error bars and the various fits in this paper indicates that other explanations involving a decreasing period, sudden jumps or even a constant period cannot be ruled out for this star and might even be equally probable. The period variations of VZ Cnc need to be regarded as uncertain at this time.

We conclude that four years after the report of the puzzling period decrease of luminous $\delta$ Scuti stars more data have become available, but that the reality of this effect is still tantalising, rather than statistically and astrophysically secure.

\section{The Delta Scuti Star Newsletter}

In order to facilitate rapid communication between researchers in this area, the present author edits a Delta Scuti Star Newsletter, which is issued regularly. Submission of news items and discussions are invited. New items can be found prior to publication on the Internet at http://venus.ast.univie.ac.at, where the contents of previous newsletters can also be accessed. 
Acknowledgments. Part of this investigation has been supported by the Austrian Fonds zur Förderung der wissenschaftlichen Forschung under Grant number S7304-AST.

\section{References}

Abt, H. A., Bollinger, G., \& Burke, E. W., Jr. 1983, ApJ, 272, 196

Arellano Ferro A., Nunez, N. S., \& Avila, J. J. 1994, PASP, 106, 696

Balona, L. A., Krisciunas, K., \& Cousins, A. W. J. 1994, MNRAS, 270, 905

Bax, J., Wehlau, W. H., \& Sharpe, S. 1995, Journ. Roy. Astron. Soc. Can., in press

Belmonte, J. A., Michel, E., Alvarez, M., et al. 1994, A\&A, 283, 121

Breger, M., 1990a, Delta Scuti Star Newsletter (Vienna), 2, 13

Breger. M. 1990b, A\&A, 240, 308

Breger, M. 1990c, ASP Conf. Ser., 11, 263

Breger, M., Garrido, R., Huang, L., et al. 1989, A\&A, 214, 209

Breger, M., Stich, J., Garrido, R., et al. 1993, A\&A, 271, 482

Breger, M., Handler, G., Serkowitsch, E., et al. 1995, A\&A, in press

Burke, E. W. Jr., Burke, E. W. III, \& Lady, S. 1977, IBVS, 1279

Cao, M., \& Jiang, S. 1992, Chin. A\&A, 16, 148

Cousins, A. W. J. 1966, Roy. Obs. Bull., 122

Dziembowski, W. A., \& Goode, P. R. 1992, ApJ, 394, 670

Dziembowski, W. A., \& Krolikowska, M. 1990, Acta Astron., 40, 19

Dziembowski, W. A., \& Pamyatnikh, A. A. 1991, A\&A, 248, L11

Frandsen, S., Viskum, M., Balona, L., \& Koen, C. 1994, in Delta Scuti Star Newsletter (Vienna), 7, 5

Goupil, M. J., Michel, E., Lebreton, et al. 1993, A\&A, 268, 546

Guzik, J. A., \& Cox, A. N. 1991, in Delta Scuti Star Newsletter (Vienna), 3, 6

Handler, G., 1994, in Delta Scuti Star Newsletter (Vienna), 7, 11

Handler, G., 1995, in Delta Scuti Star Newsletter (Vienna), 8, 1

Hildebrandt, G., Schöneich, W., \& Lange, D. 1985, Publ. Astrophys. Obs. Potsdam, 112

Kovacs, G. 1981, Acta Astron., 31, 75

Krisciunas, K., \& Guinan, E. 1990, IBVS, 3511

Kuschnig, R., Weiss, W. W., Kreidl, T. J., et al. 1990, ASP Conf. Ser., 11, 348

Lehmann, H., Scholz, G., \& Hildebrandt, G. 1995, A\&A, in press

Mantegazza, L., \& Poretti, E. 1992, A\&A, 255, 153

Mantegazza, L., \& Poretti, E. 1993, A\&A, 274, 811

Mantegazza, L., \& Poretti, E. 1994, A\&A, 281, 66

Mantegazza, L., Poretti, E., \& Zerbi, F. M. 1994, A\&A, 270, 439

Mantegazza, L., Poretti, E., \& Zerbi, F. M. 1995, A\&A, in press

Matthews, J. 1990, A\&A, 229, 452 
Michel, E., Belmonte, J. A., Alvarez, M., et al. 1992, A\&A, 255, 139

Ostermann, W. M., Breger, M., Garrido, R., et al. 1991, A\&A, 245, 543

Paparo, M., Pena, J., Peniche, R., et al. 1993, A\&A, 268, 123

Percy, J. R., Matthews, J. M., \& Wade, J. D. 1980, A\&A, 82, 172

Poretti, E., Mantegazza, L., \& Riboni, E. 1993, A\&A, 256, 113

Rodriguez, E., Lopez de Coca, P., \& Costa, V. 1995, A\&A, in press

Stobie, R. S., Pickup, D. A., \& Shobbrook, R. R. 1977, MNRAS, 179, 389

Struve, O. 1955, Sky \& Telescope, 14, 461

Sweigart, A. V., \& Renzini, A. 1979, A\&A, 71, 66

Szeidl, B., \& Mahdy, H. A. 1980, Comm. Konkoly Obs., 75, 1

Walraven, Th., Walraven, J., \& Balona, L. A. 1992, MNRAS, 254, 59

Yang, D., Jiang, S.-y., Tang, Q.-q., et al. 1993, IBVS, 3831

\section{Discussion}

Smith: I find it amusing that you discussed the new group of "slowly" variable F stars, but you did not mention the analogous group of high-degree NRP, rapidly rotating $\mathrm{F}$ giants discovered by Gordon Walker and co-workers (Walker et al. 1987, ApJ 320, L139). The co-existence of this latter class with the slow $\mathrm{F}$ variables is reminiscent of the controversy surrounding the 53 Per and "SPB" variables among the NRP B stars. This all leads me to wonder whether we are not rushing to judgement in attributing these periods as conventional $g$-modes. Perhaps there is after all room for a rotational excitation mechanism (e.g., the Lee-Saio mechanism).

Breger: I did mention these spectroscopically discovered high-degree sectorial modes, but attributed the variability to $p$-modes. In this regard they probably differ from the 53 Per or SPB stars and there seems to be no controversy. At this time the best scenario for these stars might be: (i) photometrically we see low-order, low-degree $p$-modes and possibly some low-order $g$ modes, (ii) lineprofile changes show high-degree $p$-modes and some low-degree $p$-modes, (iii) high-order $g$ modes are seen as slow variations.

Dziembowski: Mid-F slow pulsators are the likely analogue of SPB stars as Chris Waelkens has already suggested (elsewhere). Just like the metal opacity bump is responsible for $g$ mode pulsations in stars cooler than $\beta$ Cep variables, the HeII ionization bump may be responsible for $g$ mode excitation in stars cooler than $\delta$ Sct variables. Pamyatnykh and I, indeed, have found unstable high-order $g$ modes in models of mid-F stars. Unfortunately, unlike in SPB models, the convective flux is large in the driving zone; therefore we regard our results as not sufficiently reliable and we will not publish them.

Kovács: There is a general problem of using linear pulsation models for mode identification in the case of nonlinear, non-radial pulsation. The observed position of a normal mode in the frequency spectrum depends on the following factors: (i) linear non-adiabatic normal frequency; (ii) nonlinear effects; (iii) rotational frequency. For example, if 1:1 resonance is important in the case of 
a rotationally split set of modes, one can show that in the stationary nonlinear state the observed frequency splitting is equidistant, with a spacing determined by the factors mentioned above (see Goupil \& Buchler 1994).

Breger: Your important comment underscores the need to do asteroseismology of Delta Scuti stars, i.e., to detect and identify the rotationally split modes from observational data in order to compare with a variety of theoretical predictions. I would speculate that the observations will indicate even more factors than you are listing.

Frandsen: In stellar systems (open clusters etc.) the constraints on the stellar parameters are stronger. Therefore, mode identifications become much easier than for isolated stars. Also, phase studies can provide a determination of $\ell$ values that restrict the choice of frequencies to match.

Breger: You are correct. For stars in clusters such as Praesepe the distance is quite well known so that the radial order of a mode is more accurately and uniquely determined. Also, in order to obtain the important phase information, photometric measurements should be made in two colours, e.g., the $v$ and $y$ filters of the uvby system. Simultaneous spectroscopic measurements (if that could be organised) provide strong constraints.

Burki: Do you know the unbiased percentages of stable and pulsating stars in the $\delta$ Scuti region of the HR diagram? And I have the same question concerning the Ap stars (to Don Kurtz).

Breger and Kurtz: $\delta$ Scuti stars: Variability surveys yield $\sim 40 \%$ variability inside the Lower Instability Strip. Re-measurements of some 'constant' stars have found variability at the later time. This is only partially due to the nonrepetitive behaviour of $\delta$ Scuti variation. If we furthermore correct for the low incidence of variability among the classical Am stars, for "normal" stars the variability becomes $\gtrsim 75 \%$ at the $0.001 \mathrm{mag}$ level of amplitude.

Nemec: Can you give a physical explanation as to why the higher-L $\delta$ Sct stars have decreasing $P$ s and the lower-L $\delta$ Sct have increasing $P$ s?

Breger: If the observed $\dot{P}$ values are correct and not due to some irregular $P$ variations, then either the computed evolutionary tracks in this limited part of the instability strip are incorrect and the radii are actually decreasing for a while, or radial pulsation is more complicated than believed at the moment. In that case, the periods would depend strongly on the internal structure of the star. 